\title{
COMPARAÇÃO DA RESISTÊNCIA A OXIDAÇÃO DE ARAMES GALVANIZADOS POR IMERSÃO A QUENTE APLICANDO DIFERENTES CAMADAS DE CONVERSÃO A BASE DE CROMO*
}

João Vitor Mokan ${ }^{1}$ Jane Zoppas Ferreira ${ }^{2}$

\begin{abstract}
Resumo
O presente trabalho avalia o efeito das camadas de conversão a base de $\mathrm{Cr}^{\mathrm{VI}}$ e $\mathrm{Cr}^{\mathrm{III}}$ aplicadas na superfície de arames galvanizados por imersão a quente. As bitolas testadas foram 1,24mm, 1,65mm e 3,00mm, utilizando os ensaios de Névoa Salina e Câmara Úmida, avaliando o aspecto superficial dos arames após 1, 3, 6, 24 e 28 horas de exposição ao ambiente agressivo. Pode ser observado que o ensaio de Névoa Salina é pouco indicado para avaliar a oxidação superficial do arame em função de sua agressividade. Os resultados foram melhor interpretados utilizando o ensaio de Câmara Úmida. Inicialmente os arames recobertos por uma camada de conversão a base de $\mathrm{Cr}^{\text {III }}$ apresentaram desempenho levemente superior àqueles recobertos por $\mathrm{Cr}^{\mathrm{VI}}$, considerando os mesmos tempos de exposição. Após seis horas os arames galvanizados apresentaram comportamentos semelhantes. $O$ arame galvanizado com maior resistência a oxidação foi aquele protegido com $\mathrm{Cr}^{\mathrm{III}}$, porém com o passar do tempo os dois tipos de revestimento se equivalem.
\end{abstract}

Palavras-chave: Corrosão; Camadas de Conversão; Arame Galvanizado; Cromatização;

\section{COMPARISON OF THE OXIDATION RESISTANCE OF HOT DEEP GALVANIZED WIRES BY APPLYING DIFFERENT CHROMIUM CONVERSION LAYERS}

\begin{abstract}
The present work evaluates the effect of $\mathrm{Cr}^{\mathrm{VI}}$ and $\mathrm{Cr}^{\text {III }}$ conversion layers applied to the surface of hot dip galvanized wires. The tested samples wire diameters were $1.24 \mathrm{~mm}$, $1.65 \mathrm{~mm}$ and $3.00 \mathrm{~mm}$, using the Salt Spray test and Humid Atmosphere Chamber test, observing the wire surface appearance after 1, 3, 6, 24 and 28 hours of aggressive environment exposure. The surface galvanized wires surface oxidation cannot be evaluate by the Salt Spray test due to his aggressiveness. The Humid Atmosphere Chamber test promoted better results interpretations. Initially, the wires covered by a conversion layer based on $\mathrm{Cr}^{\text {III }}$ presented a slightly better performance than those covered by $\mathrm{Cr}$, considering the same times of exposure. After six hours the galvanized wires showed similar behavior. The galvanized wire with the best resistance to oxidation was the $\mathrm{Cr}^{\text {III }}$ protected, but over time, the two types of coating are equivalent.
\end{abstract}

Keywords: Corrosion; Conversion Coatings; Wire Galvanizing; Chromate.

1 Engenheiro Metalúrgico, Mestrando, Universidade Federal do Rio Grande do Sul, Porto Alegre, Rio Grande do Sul, Brasil.

2 Bacharel em Química, Doutora, Orientadora, PPGE3M, Universidade Federal do Rio Grande do Sul, Porto Alegre, Rio Grande do Sul, Brasil. 


\section{INTRODUÇÃO}

A maioria dos materiais sobre algum tipo de interação com o ambiente em que estão expostos, resultando em alguns casos, na deterioração de propriedades físicas ou simplesmente apresentando modificações de aparência [1]. Para minimizar ou retardar os efeitos provocados pela interação dos metais com o ambiente muitos recursos são utilizados. No trabalho a seguir serão comparados dois tipos de camada de conversão uma à base de cromo trivalente e outra à base de cromo hexavalente, para retardar a oxidação do Zinco, utilizado como metal de proteção do aço dos arames galvanizados a fogo em processo contínuo.

As camadas de conversão foram aplicadas em um processo de galvanização sem preparações especiais, repetindo os mesmos parâmetros com as mesmas bitolas de arames para cada condição. O objetivo foi comparar a eficiência de dois produtos em um mesmo processo, permitindo avaliar qual deles seria mais eficiente no retardo do processo de oxidação do Zinco.

As amostras foram expostas a ensaios de Câmara Úmida e Névoa Salina sendo retiradas para avaliação visual em intervalos de 1, 3, 6, 24 e 28 horas. Após avaliação visual, as amostras foram fotografadas e retornaram para o ensaio até o fim do ciclo descrito. Com os resultados foi possível gerar uma série de discussões permitindo concluir que o revestimento a base de CrIII tem uma eficiência melhor nas primeiras horas de exposição ao ambiente agressivo, equiparando-se ao revestimento de $\mathrm{Cr}^{\mathrm{VI}}$ com o passar do tempo.

\subsection{Galvanização por imersão a quente}

Galvanização por imersão a quente é o processo pelo qual uma camada de zinco é formada sobre a superfície de um aço pela imersão deste em um banho de zinco fundido [2]. O revestimento de zinco oferece uma proteção catódica ao aço à medida que o zinco se sacrifica, isto é, sofre corrosão para que o aço permaneça em sua forma metálica e assim tenha suas propriedades mantidas [4]. No processo de galvanização as primeiras etapas se constituem em preparar a superfície do aço para receber o revestimento de zinco. Assim há uma etapa de decapagem em solução de ácido clorídrico, a fim de remover a carepa dos processos anteriores e alguma oxidação presente. Após o aço passa por uma etapa de fluxagem em um banho de cloreto duplo de zinco e amônio, que prepara quimicamente a superfície do aço para as reações metalúrgicas que irão ocorrer durante a imersão no banho de zinco [4][5].Durante a imersão no banho de zinco, entre $430^{\circ} \mathrm{C}$ e $470^{\circ} \mathrm{C}$, camadas de liga ferro-zinco são formadas: a fase gama ( $($ ) , com 21 a $28 \%$ de Fe; a fase delta $(\delta)$

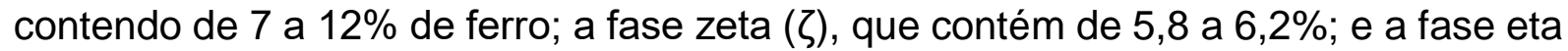
(n), constituída praticamente por zinco puro [3][5]. 


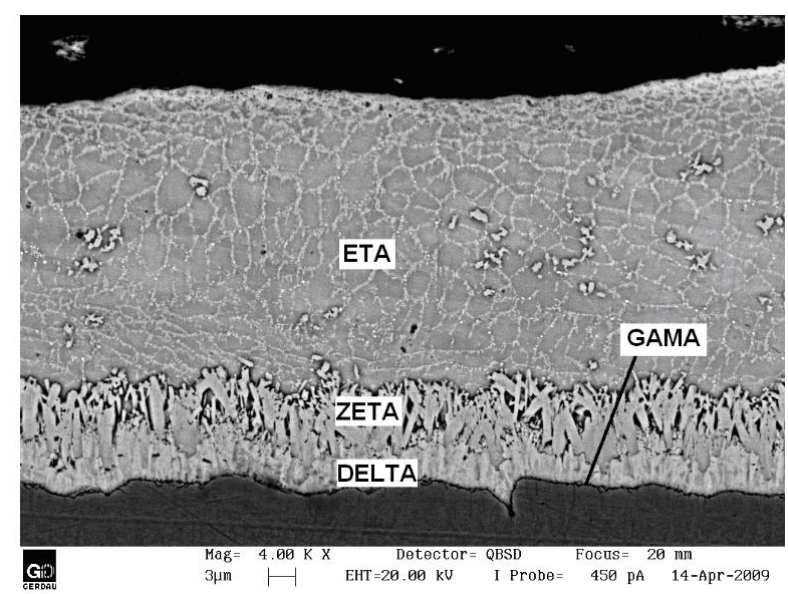

Figura 1. Imagem obtida por MEV de corte transversal de amostra de aço galvanizado [6].

A espessura da camada é controlada pelo tempo de imersão e pela velocidade de remoção da peça de dentro da cuba de zinco fundido [3]. Esses parâmetros devem ser otimizados a fim de obter uma camada homogênea e com espessura adequada segundo a aplicação. A especificação de camadas galvanizadas comumente é dada $\mathrm{em} \mathrm{g} / \mathrm{m}^{2}$. Em geral os revestimentos têm entre 20 e $30 \mathrm{~g} / \mathrm{m}^{2}$, que corresponde a uma espessura de 10 a $30 \mu \mathrm{m}$ [4]. A vida útil da camada galvanizada é diretamente proporcional à sua espessura, dada uma constante de proporcionalidade que depende do meio ambiente à que o aço galvanizado será exposto [5]. A galvanização é um sistema com boa resistência à corrosão, sendo essa a principal razão de seu emprego, representando mais da metade do uso mundial de zinco [3]. O zinco é capaz de formar uma camada auto protetora quando exposto a atmosferas não poluídas. Há a formação de óxido de zinco, $\mathrm{ZnO}$, ou hidróxido de zinco, $\mathrm{Zn}(\mathrm{OH})_{2}$, que sob ação do gás carbônico presente na atmosfera forma um carbonato básico de zinco insolúvel, $3 \mathrm{Zn}(\mathrm{OH})_{2} \cdot \mathrm{ZnCO}_{3}$, ou seja, uma camada passiva [3] [5]. Porém em atmosferas poluídas, principalmente com produtos ácidos, como óxidos de enxofre, o zinco sofre severa corrosão [3]. Em ambientes extremamente úmidos pode haver formação de produto de corrosão branca [4]. Assim, alguns tratamentos superficiais são aplicados ao aço galvanizado para melhorar a durabilidade da camada de zinco, como aplicação de revestimentos de conversão, como as cromatizações hexavalente e trivalente.

\subsection{Revestimentos de conversão}

Um revestimento de conversão é uma camada inorgânica formada sobre a superfície de um metal a partir da imersão deste em uma solução aquosa contendo os íons que se deseja precipitar sobre a superfície. Durante o processo de conversão a camada de óxido natural na superfície do metal é dissolvida e os demais íons da solução iniciam o crescimento do revestimento de conversão [7]. Um dos processos de conversão mais comuns no meio industrial é a cromatização. Essa técnica é amplamente usada graças às propriedades anticorrosivas da camada convertida e à relativa facilidade de controle de processo. Porém a utilização de cromo hexavalente vem sendo restringida devido à sua toxicidade e efeito carcinogênico, o que incentiva a pesquisa por novos processos de conversão que sejam ambientalmente amigáveis. 


\subsection{Cromatização}

Cromatização é um processo de conversão de superfícies por soluções contendo cromatos ou ácido crômico [3]. Esse revestimento pode ser aplicado a superfícies metálicas ou sobre camadas de óxidos e fosfatos e tem o objetivo de aumentar a resistência à corrosão das superfícies, selar poros e melhorar a pintabilidade. As formulações podem conter anidrido crômico, dicromato de sódio, dicromato de potássio, cromato de sódio, ácidos orgânicos, ácido sulfúrico e clorídrico, e ativadores como sulfato, nitrato, cloreto, fluoreto, fosfato ou acetato, que aceleram o ataque ao metal. O hidrogênio resultante reduz parte do íon cromato, formando hidróxido de cromo e cromato básico de cromo, $\mathrm{Cr}(\mathrm{OH})_{3} \cdot \mathrm{Cr}(\mathrm{OH}) \mathrm{CrO}_{4}$, que se depositam sobre a superfície, na forma de um gel [3]. As reações possíveis durante o processo de cromatização são:

-Dissolução do metal:

$\mathrm{M} \rightarrow \mathrm{M}^{\mathrm{n}+}+\mathrm{ne}$

$2 \mathrm{H}^{+}+2 \mathrm{e} \rightarrow \mathrm{H}_{2}$

-Redução do íon cromato:

$\mathrm{HCr}_{2} \mathrm{O}_{7}^{-}+3 \mathrm{H}_{2} \rightarrow 2 \mathrm{Cr}(\mathrm{OH})_{3}+\mathrm{OH}^{-}$

-Elevação do pH:

$\mathrm{HCr}_{2} \mathrm{O}_{7}^{-}+\mathrm{H}_{2} \mathrm{O} \rightarrow 2 \mathrm{CrO}_{4}^{2-}+3 \mathrm{H}^{+}$

$3 \mathrm{H}^{+}+\mathrm{H}_{2} \mathrm{O}+4 \mathrm{e} \rightarrow 2 \mathrm{H}_{2}{ }^{\lambda}+\mathrm{OH}^{-}$

-Formação do gel:

$2 \mathrm{Cr}(\mathrm{OH})_{3}+\mathrm{CrO}_{4}{ }^{2-}+2 \mathrm{H}^{+} \rightarrow \mathrm{Cr}(\mathrm{OH})_{3} \cdot \mathrm{CrOH} \cdot \mathrm{CrO}_{4} \downarrow+2 \mathrm{H}_{2} \mathrm{O}$

$\mathrm{Cr}_{2} \mathrm{O}_{7}^{2-}+8 \mathrm{H}^{+}+6 \mathrm{e} \rightarrow \mathrm{Cr}_{2} \mathrm{O}_{3}+4 \mathrm{H}_{2} \mathrm{O}$

A cromatização pode ser aplicada sobre alumínio, magnésio, cádmio, aço, zinco entre outros. O processo é feito em temperatura ambiente e o tempo de tratamento varia de alguns segundos a minutos, a depender da espessura desejada para a camada convertida. Em geral para aços galvanizados o tempo de imersão fica entre 15 e 60s. A coloração final obtida pela superfície depende da espessura do revestimento, que fica entre 0,01 a $1 \mu \mathrm{m}$, dando muitas vezes um aspecto iridescente à peça. $\mathrm{O}$ revestimento pode ser aplicado por imersão ou spray e deve ser cuidadosamente lavado e seco [3]. A maioria dos filmes de cromato são macios e gelatinosos assim que formados. Uma vez secos eles vagarosamente se tornam mais duros e se tornam hidrofóbicos, menos solúveis e mais resistentes à abrasão com o envelhecimento. Eles oferecem excelente resistência à corrosão devido à barreira física que o revestimento inorgânico oferece e ao efeito inibidor de corrosão do cromo hexavalente presente no filme [8]. Mesmo que o filme sofra algum dano mecânico ele ainda mantém boas propriedades anticorrosivas porque o teor de cromo hexavalente é 
lentamente lixiviado, e reagindo com a umidade e restaurando o filme de óxido de cromo (efeito self-healing) [9]. A quantidade de cromo hexavalente presente no filme pode ser um indicador da proteção contra corrosão oferecida pelo revestimento, assim como a espessura total do filme [8]. A Figura 2 apresenta uma imagem da camada cromatiza evidenciando fissuras típicas que, pelo efeito self-healing do cromo hexavalente mantém por mais tempo as características anticorrosivas [10]. Essas vantagens têm feito deste tratamento um método padrão para proteção contra corrosão para diversas ligas ferrosas e não ferrosas. Entretanto, o cromo hexavalente é uma substância tóxica devido ao seu efeito carcinogênico e ambientalmente perigoso [2] [11].

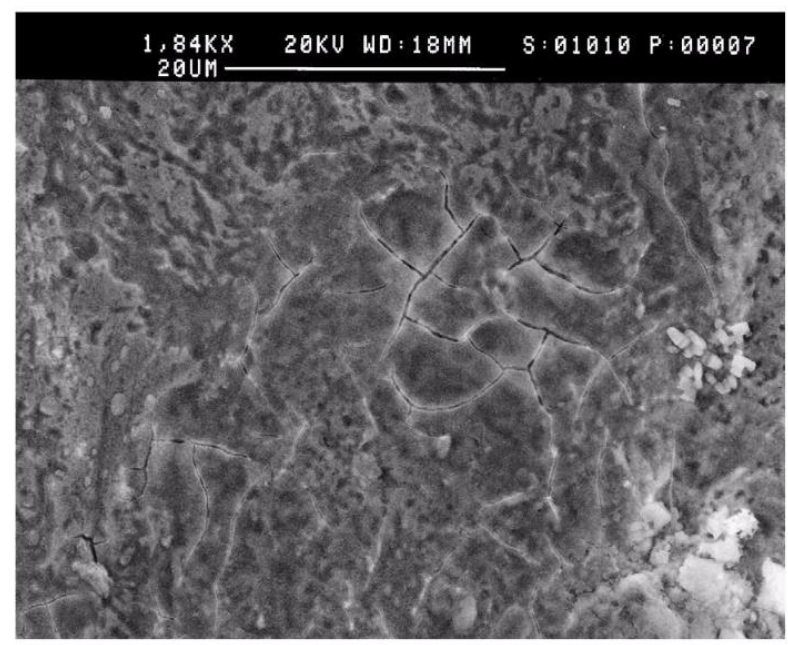

Figura 2. Imagem de MEV de cromatização hexavalente sobre aço galvanizado, as fissuras são típicas da camada de cromato [10].

Devido ao problema ambiental e de saúde que a cromatização hexavalente pode gerar caso os cuidados no processamento, manuseio e descarte dos resíduos e peças não forem observados, revestimentos de conversão utilizando o cromo trivalente passaram a ser uma alternativa ao tratamento convencional. $O$ cromo trivalente é espécie intermediária no processo de cromatização hexavalente e forma a camada de óxido crômico sobre a superfície metálica [12]. Possui toxicidade muito inferior ao hexavalente e é metabolizado pelo organismo humano [13]. As soluções de cromatização trivalente podem conter nitrato como oxidante e hidrofosfato de sódio como complexante, para estabilização dos íons de $\mathrm{Cr}^{3+}$ na solução. O tempo de imersão da amostra na solução é determinante para resistência à corrosão. Um tempo insuficiente leva à formação de uma camada fina com proteção limitada. No entanto, um tempo muito longo promove fissuras na camada cromatizada, permitindo a penetração de eletrólito e a dissolução do metal de base, deteriorando as propriedades de resistência à corrosão [12]. As etapas do processo de cromatização trivalente envolvem uma ativação do substrato metálico em solução de ácido nítrico antes da imersão na solução de conversão, cujas formulações podem conter nitrato de sódio, nitrato de cromo [10] [12]. O pH das soluções deve ficar entre 1,7 e 2,4 [14] para uma melhor dissolução do metal do substrato e consequente deposição da camada de conversão. O tempo de imersão pode variar entre 15 e 60s [12]. A temperatura durante a deposição deve ser mantida entre 50 e $60^{\circ} \mathrm{C}$ [10] [14]. As reações propostas para o processo de conversão do cromo trivalente sobre o zinco são [12]: 
-Reação anódica:

$$
\mathrm{Zn} \rightarrow \mathrm{Zn}^{2+}+2 \mathrm{e}
$$

-Reação catódica:

$2 \mathrm{H}^{+}+2 \mathrm{e} \rightarrow \mathrm{H}_{2}$

A redução dos íons de hidrogênio leva ao aumento do $\mathrm{pH}$ nas proximidades da superfície metálica, que resulta na precipitação de hidróxido de cromo.

$$
\mathrm{Cr}_{3}^{+}+3 \mathrm{OH}^{-} \rightarrow \mathrm{Cr}(\mathrm{OH})_{3}
$$

A camada convertida funciona como barreira, dificultando o transporte de oxigênio até o substrato metálico e retardando a dissolução do zinco. Entretanto uma vez que a camada for danificada, ela não é capaz de se regenerar, como ocorre na cromatização hexavalente [12][15]. Tanto a cromatização hexavalente como a trivalente são gelatinosas assim que depositadas. Durante a secagem da camada é comum o aparecimento de fissuras devido a tensões internas do revestimento. No caso da cromatização trivalente que não possui a habilidade de se auto regenerar, essas fissuras podem acelerar a degradação das propriedades anticorrosivas da camada [15]. O uso de $\mathrm{Co}^{2+}$ e $\mathrm{Ni}^{2+}$ nas soluções de cromatização trivalente foi reportado como um melhorador das propriedades anticorrosivas da camada convertida, pois estes íons se depositam na forma de hidróxidos nas fissuras formadas após a secagem do filme [15]. Mesmo assim, a cromatização trivalente fornece proteção contra corrosão inferior à cromatização hexavalente.

\section{MATERIAIS E MÉTODOS}

Foram utilizados para testar as diferentes camadas de conversão os arames galvanizados por imersão a quente de bitolas $1,24 \mathrm{~mm}, 1,65 \mathrm{~mm}$ e $3,00 \mathrm{~mm}$, imersos em dois banhos, um à base de cromo hexavalente e outro à base de cromo trivalente, durante um determinado tempo resultante da velocidade de produção de cada um dos fios. O tanque possuía comprimento de imersão de $450 \mathrm{~mm}$, Figura 3 . Antes de cada teste foi realizada a lavagem do equipamento parta então montar o banho de teste. Para avaliar a resistência a oxidação de cada arame, foram utilizados os ensaios de Câmara Úmida e Névoa Salina.

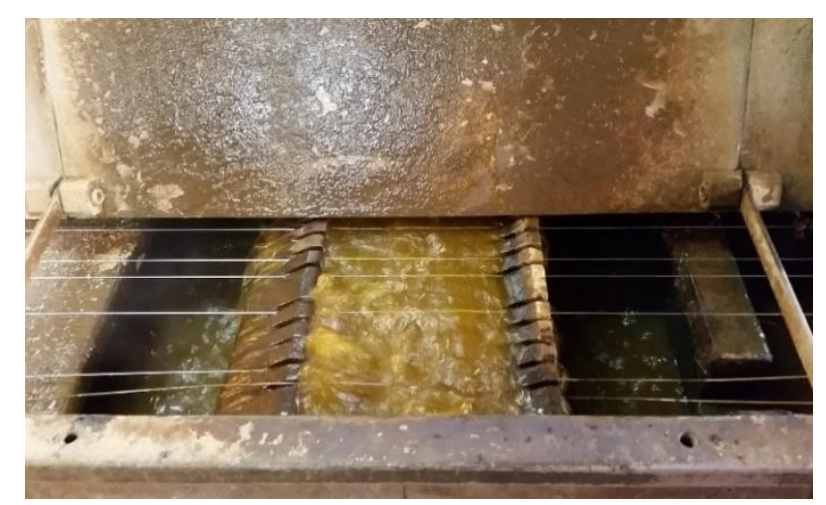

Figura 3: Tanque utilizado para confecção e aplicação dos banhos de conversão. 
- ENSAIO DE CORROSÃO POR EXPOSIÇÃO À ATMOSFERA ÚMIDA SATURADA DE REVESTIMENTOS, SEGUNDO ABNT NBR 8095 E ISO 6270-2 (Atmosfera CH), em equipamento com volume de 300 litros, na temperatura de $40^{\circ} \mathrm{C}+/-3^{\circ} \mathrm{C}$, com umidade relativa de $100 \%$. Após cada ciclo de ensaio as amostras foram secas, examinadas e fotografadas.

- ENSAIO DE CORROSÃO POR EXPOSIÇÃO À NÉVOA SALINA, SEGUNDO ABNT NBR 8094 E ASTM B 117, em equipamento com volume de 1000 litros, na temperatura de $35^{\circ} \mathrm{C} \pm 2^{\circ} \mathrm{C}$, Concentração $\mathrm{NaCl}$ de $50 \pm 5 \mathrm{~g} / \mathrm{l}$, pH solução de $6,5-7,2$, Pressão do ar de $0,7-1,4 \mathrm{kgf} / \mathrm{cm}^{2}$, Volume condensado para $80 \mathrm{~cm}^{2}$ de $1,5 \pm 0,5 \mathrm{ml} / \mathrm{h}$. As amostras foram limpas com água corrente e secas ao final de cada ciclo de ensaio. Os cromatizantes utilizados foram:

- Parcolene 60A, fabricante Henkel, aplicado a temperatura ambiente e pH de 3,5 a 4,5 , segundo orientação do fabricante.

- Surtec 541V, fabricante Surtec, na concentração de $10 \mathrm{ml} / \mathrm{l}$ aplicado a temperatura ambiente, com pH limite de 5,6, segundo orientação do fabricante.

As amostras foram avaliadas visualmente seguindo padrão de codificação de acordo com a Tabela 1. Os intervalos de exposição foram de 1, 3, 6, 24 e 28 horas.

Tabela 1: codificação da avaliação visual das amostras

\begin{tabular}{|ll|ll|}
\hline \multicolumn{2}{|c|}{ Aspecto ou Acabamento } & \multicolumn{2}{c|}{ Graus de Corrosão } \\
\hline B-Branco & CR - Cromatizado & 0-Perfeito & 3 -Áreas localizadas \\
V-Vermelho & Vd-Verde & 1- Pontos em áreas localizadas & 4- Parcial : Pontos e Áreas \\
\hline
\end{tabular}

Inicialmente foram comparados os arames galvanizados com aplicação da camada de conversão a base de cromo hexavalente e sem nenhum tipo de tratamento. Em seguida foram comparados os arames galvanizados com aplicação de camadas de conversão a base de cromo hexavalente e trivalente.

\section{RESULTADOS E DISCUSSÃO}

Cada arame galvanizado permaneceu no banho com a solução de conversão de acordo com os tempos informados na Tabela 2. Os tempos foram inferiores aos citados na literatura para a formação de um filme espesso suficiente para uma boa proteção, porém retrataram a realidade do processo de produção. Os filmes formados, em função do tempo, provavelmente eram de fina espessura.

Tabela 2: Tempo de permanência do tanque com solução

\begin{tabular}{|c|c|}
\hline Amostra & Tempo (s) \\
\hline 1,24 & 0,38 \\
\hline 1,65 & 0,30 \\
\hline 3,00 & 0,45 \\
\hline
\end{tabular}

A primeira etapa do trabalho consistiu em avaliar a resistência a corrosão dos arames galvanizados sem a camada de conversão e com a camada a base de cromo hexavalente através de dois tipos de ensaio, Névoa Salina e Câmara Úmida. Na Tabela 3, no ensaio de Névoa Salina, a bitola de menor diâmetro apresentou corrosão 
menos severa nas primeiras três horas (B1), o arame galvanizado de $3,00 \mathrm{~mm}$ já apresentava corrosão branca em áreas localizadas (B3). Com seis horas de exposição o efeito protetivo da camada de conversão hexavalente foi percebido nos arames finos (1,24 e 1,65mm), eles apresentaram corrosão apenas em pontos em áreas localizadas (B1), enquanto os arames galvanizados revestidos apenas com zinco já estavam com corrosão branca em áreas localizadas (B3). Após vinte quatro horas, todos os corpos de prova expostos a atmosfera salina, apresentaram corrosão em pontos e áreas localizadas (B4).

Ainda na Tabela 2, no ensaio de Câmara Úmida, foi percebido que o ensaio é menos agressivo permitindo acompanhar a evolução da corrosão superficial com maior detalhe. Após seis horas de exposição a atmosfera úmida, foi percebido a corrosão em áreas localizadas na amostra de arame galvanizado $1,65 \mathrm{~mm}$, enquanto, as bitolas 1,24 e $3,00 \mathrm{~mm}$ se mostraram significativamente protegidas contra a corrosão, conflitando em partes com o resultado observado no ensaio de Névoa Salina. A bitola $1,24 \mathrm{~mm}$ continuou apresentando uma pequena vantagem sobre as demais bitolas. Com vinte quatro horas de exposição o nível de corrosão em todos corpos de prova ainda estava restrito a áreas localizadas (B3). O nível de oxidação parcial (B4) foi atingido após vinte oito horas de exposição.

Nas primeiras horas de ensaio, em ambos os métodos, observou-se pontos de corrosão em áreas localizadas dos corpos de prova (B1).

Tabela 3: Ensaio comparando os arames galvanizados com camada de reversão a base de cromo hexavalente e sem camada de reversão.

\begin{tabular}{|c|c|c|c|c|c|c|c|c|c|c|c|c|c|c|}
\hline \multirow{3}{*}{$\begin{array}{l}\text { Bitola } \\
(\mathrm{mm})\end{array}$} & \multicolumn{7}{|c|}{ Névoa Salina } & \multicolumn{7}{|c|}{ Câmara Úmida } \\
\hline & \multirow{2}{*}{ Amostra } & \multirow{2}{*}{ Revestimento } & \multicolumn{5}{|c|}{ Tempo (h) } & \multirow{2}{*}{ Amostra } & \multirow{2}{*}{ Revestimento } & \multicolumn{5}{|c|}{ Tempo (h) } \\
\hline & & & 1 & 3 & 6 & 24 & 28 & & & 1 & 3 & 6 & 24 & 28 \\
\hline \multirow{6}{*}{3,00} & A1 & \multirow{3}{*}{$\mathrm{Zn}+\mathrm{Cr}^{\mathrm{VI}}$} & BO & B1 & B3 & B4 & B4 & A9 & \multirow{3}{*}{$\mathrm{Zn}+\mathrm{Cr}^{\mathrm{VI}}$} & BO & B1 & B1 & B3 & B4 \\
\hline & $\mathrm{A} 2$ & & B0 & B1 & B3 & B4 & B4 & A10 & & BO & B1 & B1 & B3 & B4 \\
\hline & A5 & & BO & B1 & B3 & B4 & B4 & A11 & & BO & B1 & B1 & B3 & B4 \\
\hline & A3 & \multirow{3}{*}{$\mathrm{Zn}$} & BO & B3 & B3 & B4 & B4 & A7 & \multirow{3}{*}{$\mathrm{Zn}$} & $\mathrm{BO}$ & B3 & B3 & B3 & B4 \\
\hline & A4 & & BO & B3 & B3 & B4 & B4 & A8 & & BO & B3 & B3 & B3 & B4 \\
\hline & A6 & & BO & B3 & B3 & B4 & B4 & $\mathrm{A} 12$ & & BO & B3 & B3 & B3 & B4 \\
\hline \multirow{6}{*}{1,65} & B3 & \multirow{3}{*}{$\mathrm{Zn}+\mathrm{Cr}^{\mathrm{VI}}$} & BO & B1 & B1 & B4 & B4 & B7 & \multirow{3}{*}{$\mathrm{Zn}+\mathrm{Cr}^{\mathrm{VI}}$} & $\mathrm{BO}$ & B1 & B3 & B3 & B4 \\
\hline & B4 & & BO & B1 & B1 & B4 & B4 & B8 & & $\mathrm{BO}$ & B1 & B3 & B3 & B4 \\
\hline & B6 & & BO & B1 & B1 & B4 & B4 & B12 & & $\mathrm{BO}$ & B1 & B3 & B3 & B4 \\
\hline & B1 & \multirow{3}{*}{$\mathrm{Zn}$} & BO & B1 & B3 & B4 & B4 & B9 & \multirow{3}{*}{$\mathrm{Zn}$} & BO & B1 & B3 & B3 & B4 \\
\hline & B2 & & BO & B1 & B3 & B4 & B4 & B10 & & BO & B1 & B3 & B3 & B4 \\
\hline & B5 & & BO & B1 & B3 & B4 & B4 & B11 & & BO & B1 & B3 & B3 & B4 \\
\hline \multirow{6}{*}{1,24} & $\mathrm{C} 2$ & \multirow{3}{*}{$\mathrm{Zn}+\mathrm{Cr}^{\mathrm{VI}}$} & BO & B1 & B1 & B4 & B4 & $\mathrm{C} 2$ & \multirow{3}{*}{$\mathrm{Zn}+\mathrm{Cr}^{\mathrm{VI}}$} & BO & B1 & B1 & B3 & B4 \\
\hline & $\mathrm{C3}$ & & BO & B1 & B1 & B4 & B4 & $\mathrm{C} 3$ & & BO & B1 & B1 & B3 & B4 \\
\hline & C6 & & BO & B1 & B1 & B4 & B4 & C6 & & BO & B1 & B1 & B3 & B4 \\
\hline & $\mathrm{C} 1$ & \multirow{3}{*}{$\mathrm{Zn}$} & BO & B1 & B3 & B4 & B4 & $\mathrm{C} 1$ & \multirow{3}{*}{$\mathrm{Zn}$} & BO & B1 & B1 & B3 & B4 \\
\hline & $\mathrm{C4}$ & & BO & B1 & B3 & B4 & B4 & $\mathrm{C4}$ & & BO & B1 & B1 & B3 & B4 \\
\hline & $\mathrm{C5}$ & & BO & B1 & B3 & B4 & B4 & $\mathrm{C5}$ & & BO & B1 & B1 & B3 & B4 \\
\hline
\end{tabular}

Após comprovar a efetividade das camadas de conversão, a Tabela 4 permite avaliar a resistência das camadas de conversão hexavalente e trivalente. Foi observado na 
primeira hora de ensaio que o revestimento a base de cromo trivalente obteve um desempenho melhor em relação ao revestimento de cromo hexavalente. A partir da terceira hora a vantagem foi mais discreta. Com o passar do tempo a eficiência dos dois revestimentos foram semelhantes.

Tabela 4: Comparativo das camadas de conversão avaliadas pelo ensaio de Névoa Salina.

\begin{tabular}{|c|c|c|c|c|c|c|c|c|c|c|c|c|}
\hline \multirow{3}{*}{$\begin{array}{l}\text { Bitola } \\
(\mathrm{mm})\end{array}$} & \multicolumn{12}{|c|}{ Névoa Salina } \\
\hline & \multirow{2}{*}{ Amostra } & \multirow{2}{*}{ Revestimento } & \multicolumn{4}{|c|}{ Tempo (h) } & \multirow{2}{*}{ Amostra } & \multirow{2}{*}{ Revestimento } & \multicolumn{4}{|c|}{ Tempo (h) } \\
\hline & & & 1 & 3 & 6 & 24 & & & 1 & 3 & 6 & 24 \\
\hline \multirow{10}{*}{3,00} & A1 & \multirow{10}{*}{$\mathrm{Cr}^{\prime \prime \prime}$} & B3 & B3 & B4 & B5 & A31 & \multirow{10}{*}{$\mathrm{Cr}^{\mathrm{IV}}$} & B4 & B4 & B5 & B5 \\
\hline & $\mathrm{A} 2$ & & B3 & B3 & B4 & B5 & A32 & & B4 & B4 & B5 & B5 \\
\hline & A3 & & B3 & B3 & B4 & B5 & A33 & & B4 & B4 & B5 & B5 \\
\hline & A4 & & B3 & B3 & B4 & B5 & A34 & & B4 & B4 & B5 & B5 \\
\hline & A5 & & B3 & B3 & B4 & B5 & A35 & & B4 & B4 & B5 & B5 \\
\hline & A6 & & B3 & B3 & B4 & B5 & A36 & & B4 & B4 & B5 & B5 \\
\hline & A7 & & B3 & B3 & B4 & B5 & A37 & & B4 & B4 & B5 & B5 \\
\hline & A8 & & B3 & B3 & B4 & B5 & A38 & & B4 & B4 & B5 & B5 \\
\hline & A9 & & B3 & B3 & B4 & B5 & A39 & & B4 & B4 & B5 & B5 \\
\hline & A10 & & B3 & B3 & B4 & B5 & A40 & & B4 & B4 & B5 & B5 \\
\hline \multirow{10}{*}{1,65} & A11 & \multirow{10}{*}{$\mathrm{Cr}^{\prime \prime \prime}$} & B1 & B3 & B4 & B5 & A41 & \multirow{10}{*}{$\mathrm{Cr}^{\mathrm{IV}}$} & B4 & B4 & B4 & B5 \\
\hline & $\mathrm{A} 12$ & & B1 & B3 & B4 & B5 & A42 & & B4 & B4 & B4 & B5 \\
\hline & A13 & & B1 & B3 & B4 & B5 & A43 & & B4 & B4 & B4 & B5 \\
\hline & A14 & & B1 & B3 & B4 & B5 & A44 & & B4 & B4 & B4 & B5 \\
\hline & A15 & & B1 & B3 & B4 & B5 & A45 & & B4 & B4 & B4 & B5 \\
\hline & A16 & & B1 & B3 & B4 & B5 & A46 & & B4 & B4 & B4 & B5 \\
\hline & A17 & & B1 & B3 & B4 & B5 & A47 & & B4 & B4 & B4 & B5 \\
\hline & A18 & & B1 & B3 & B4 & B5 & A48 & & B4 & B4 & B4 & B5 \\
\hline & A19 & & B1 & B3 & B4 & B5 & A49 & & B4 & B4 & B4 & B5 \\
\hline & $\mathrm{A} 20$ & & B1 & B3 & B4 & B5 & $\mathrm{A} 50$ & & B4 & B4 & B4 & B5 \\
\hline \multirow{10}{*}{1,24} & A21 & \multirow{10}{*}{$\mathrm{Cr}^{\prime \prime \prime \prime}$} & B1 & B3 & B4 & B5 & A51 & \multirow{10}{*}{$\mathrm{Cr}^{\mathrm{IV}}$} & B3 & B3 & B4 & B5 \\
\hline & $\mathrm{A} 22$ & & B1 & B3 & B4 & B5 & A52 & & B3 & B3 & B4 & B5 \\
\hline & A23 & & B1 & B3 & B4 & B5 & A53 & & B3 & B3 & B4 & B5 \\
\hline & $\mathrm{A} 24$ & & B1 & B3 & B4 & B5 & A54 & & B3 & B3 & B4 & B5 \\
\hline & A25 & & B1 & B3 & B4 & B5 & A55 & & B3 & B3 & B4 & B5 \\
\hline & A26 & & B1 & B3 & B4 & B5 & A56 & & B3 & B3 & B4 & B5 \\
\hline & $\mathrm{A} 27$ & & B1 & B3 & B4 & B5 & A57 & & B3 & B3 & B4 & B5 \\
\hline & $\mathrm{A} 28$ & & B1 & B3 & B4 & B5 & A58 & & B3 & B3 & B4 & B5 \\
\hline & A29 & & B1 & B3 & B4 & B5 & A59 & & B3 & B3 & B4 & B5 \\
\hline & $\mathrm{A} 30$ & & B1 & B3 & B4 & B5 & $\mathrm{A} 60$ & & B3 & B3 & B4 & B5 \\
\hline
\end{tabular}

$\mathrm{Na}$ Tabela 5, as camadas de conversão foram testadas utilizando no ensaio de Câmara Úmida. Os arames tratados com a camada de conversão trivalente tiveram desempenho superior aos arames tratados com camada hexavalente. Nesta avaliação ficou claro que a bitola 3,00 mm apresenta maior dificuldade para resistir a corrosão, apresentando menor eficiência de seu revestimento protetor, talvez por chegar ao 
banho de conversão com maior temperatura que as demais em função da menor taxa de resfriamento. Com a temperatura maior a formação da camada de reversão fica prejudicada.

Tabela 5: Comparativo das camadas de conversão avaliadas pelo ensaio de Câmara Úmida.

\begin{tabular}{|c|c|c|c|c|c|c|c|c|c|c|c|c|}
\hline \multirow{3}{*}{$\begin{array}{l}\text { Bitola } \\
(\mathrm{mm})\end{array}$} & \multicolumn{12}{|c|}{ Câmara Úmida } \\
\hline & \multirow{2}{*}{ Amostra } & \multirow{2}{*}{ Revestimento } & \multicolumn{4}{|c|}{ Tempo (h) } & \multirow{2}{*}{ Amostra } & \multirow{2}{*}{ Revestimento } & \multicolumn{4}{|c|}{ Tempo (h) } \\
\hline & & & 1 & 3 & 6 & 24 & & & 1 & 3 & 6 & 24 \\
\hline \multirow{10}{*}{3,00} & A1 & \multirow{10}{*}{$\mathrm{Cr}^{\mathrm{III}}$} & BO & BO & B3 & B4 & A31 & \multirow{10}{*}{$\mathrm{Cr}^{\mathrm{IV}}$} & BO & B3 & B3 & B4 \\
\hline & $\mathrm{A} 2$ & & BO & BO & B3 & B4 & A32 & & BO & B3 & B3 & B4 \\
\hline & A3 & & BO & BO & B3 & B4 & A33 & & BO & B3 & B3 & B4 \\
\hline & A4 & & BO & BO & B3 & B4 & A34 & & BO & B3 & B3 & B4 \\
\hline & A5 & & BO & BO & B3 & B4 & A35 & & BO & B3 & B3 & B4 \\
\hline & A6 & & BO & BO & B3 & B4 & A36 & & BO & B3 & B3 & B4 \\
\hline & A7 & & BO & BO & B3 & B4 & A37 & & BO & B3 & B3 & B4 \\
\hline & A8 & & B0 & BO & B3 & B4 & A38 & & BO & B3 & B3 & B4 \\
\hline & A9 & & BO & BO & B3 & B4 & A39 & & BO & B3 & B3 & B4 \\
\hline & $\mathrm{A} 10$ & & $\mathrm{~B} 0$ & BO & B3 & B4 & $\mathrm{A} 40$ & & BO & B3 & B3 & B4 \\
\hline \multirow{10}{*}{1,65} & A11 & \multirow{10}{*}{$\mathrm{Cr}^{\mathrm{III}}$} & BO & BO & B1 & B3 & A41 & \multirow{10}{*}{$\mathrm{Cr}^{\mathrm{IV}}$} & BO & B1 & B3 & B4 \\
\hline & A12 & & $\mathrm{BO}$ & BO & B1 & B3 & A42 & & BO & B1 & B3 & B4 \\
\hline & A13 & & $\mathrm{B} 0$ & BO & B1 & B3 & A43 & & BO & B1 & B3 & B4 \\
\hline & A14 & & BO & BO & B1 & B3 & A44 & & BO & B1 & B3 & B4 \\
\hline & A15 & & BO & BO & B1 & B3 & A45 & & BO & B1 & B3 & B4 \\
\hline & A16 & & $\mathrm{B} 0$ & BO & B1 & B3 & A46 & & BO & B1 & B3 & B4 \\
\hline & A17 & & BO & BO & B1 & B3 & A47 & & BO & B1 & B3 & B4 \\
\hline & A18 & & BO & BO & B1 & B3 & A48 & & BO & B1 & B3 & B4 \\
\hline & A19 & & BO & BO & B1 & B3 & A49 & & BO & B1 & B3 & B4 \\
\hline & A20 & & B0 & B0 & B1 & B3 & A50 & & BO & B1 & B3 & B4 \\
\hline \multirow{10}{*}{1,24} & $\mathrm{~A} 21$ & \multirow{10}{*}{$\mathrm{Cr}^{\prime \prime \prime \prime}$} & $\mathrm{BO}$ & BO & B1 & B3 & A51 & \multirow{10}{*}{$\mathrm{Cr}^{\mathrm{IV}}$} & BO & B1 & B3 & B3 \\
\hline & $\mathrm{A} 22$ & & BO & BO & B1 & B3 & A52 & & BO & B1 & B3 & B3 \\
\hline & A23 & & BO & BO & B1 & B3 & A53 & & BO & B1 & B3 & B3 \\
\hline & A24 & & $\mathrm{B} 0$ & BO & B1 & B3 & A54 & & BO & B1 & B3 & B3 \\
\hline & A25 & & BO & BO & B1 & B3 & A55 & & BO & B1 & B3 & B3 \\
\hline & A26 & & BO & BO & B1 & B3 & A56 & & BO & B1 & B3 & B3 \\
\hline & $\mathrm{A} 27$ & & $\mathrm{BO}$ & BO & B1 & B3 & A57 & & BO & B1 & B3 & B3 \\
\hline & A28 & & BO & BO & B1 & B3 & A58 & & BO & B1 & B3 & B3 \\
\hline & A29 & & BO & BO & B1 & B3 & A59 & & BO & B1 & B3 & B3 \\
\hline & A30 & & BO & BO & B1 & B3 & A60 & & BO & B1 & B3 & B3 \\
\hline
\end{tabular}

\section{CONCLUSÃo}

- O ensaio de Câmara Úmida permite uma melhor observação da evolução da corrosão nos arames galvanizados, principalmente quando se deseja observar o efeito apenas da corrosão sobre a superfície do arame (corrosão branca); 
- Como passar do tempo, a evolução do processo de corrosão é maior nos arames sem a camada de reversão expostos aos ensaios de Névoa Salina e Câmara Úmida, essencialmente devido a formação da camada de conversão que promove maior resistência a corrosão;

- Pode-se afirmar que o filme de proteção formado pelo cromo trivalente inicialmente apresentou melhor resultado que o filme formado pelo cromo hexavalente, resultado contrário ao esperado e comprovado em alguns trabalhos utilizando métodos de laboratório;

- O tempo de permanência das amostras em solução é pequeno quando comparado a indicação mínima observada na literatura que é de quinze segundos, prejudicando a formação de um filme resistente a corrosão.

\section{REFERÊNCIAS}

1 CALLISTER, William D. Jr. Ciência e Engenharia de Materiais: Uma Introdução. Quinta Edição. Rio de Janeiro: LTC,2002.

2 ROBERGE, P. R.Handbook of Corrosion Engineering. s.l.: McGraw-Hill, 2012.

3 GENTIL, V. Corrosão. 6a ed. Rio de Janeiro: LTC, 2012.

4 CARTER, V. E.Metallic Coatings for Corrosion Control. 1 $1^{\text {a }}$ ed. London: NewnesButterworths, 1977.

5 GARCIA Jr., L. e MALLET, A.Curso de Galvanização do Grupo Gerdau. 1993.

6 CAMPANHER, C. G.Otimização experimental em processo de galvanização de arames de camada pesada. Dissertação de Mestrado. Porto Alegre: UFRGS, 2009.

7 STROMBERG, C., et al., et al. Synthesis and characterization of surface gradient thin conversion films on zinc coated steel. Electrochimica Acta. 2006, Vol. 52, pp. 804-815.

8 EPPENSTEINER, F. W e JENKINS, M. R. Chromate conversion coatings. Metal Finishing. 1999, Vol. 97, pp. 494-506.

9 PAUSSA, L., ANDREATTA, F. e ALDIGHIERI, P. E FEDRIZZI, L.Critical aspects in the electrochemical study of unstable coated metallic substrates. Progress in Organic Coatings. 2010, Vol. 69, pp. 225-234.

10 DEFLORIAN, F., et al., et al. EIS study of organic coating on zinc surface pretreated with environmentally friendly products. Progress in Organic Coatings. 2005, Vol. 52, pp. 271-279.

11 HAMDY, A. S. E. e FARAHAT, M.Chrome-free zirconia-based protective coatings for magnesium alloys. Surface \& Coatings Technology. 2010, Vol. 204, pp. 2834-2840.

12 CHANG, Y. T., et al., et al. The effects of immersion time on morphology and electrochemical properties of the $\mathrm{Cr}$ (III)-based conversion coatings on zinc coated steel surface. Corrosion Science. 2008, Vol. 50, pp. 3494-3499.

13 SREEKANTH, R., PATTABHI, V. e RAJAN, S. S.Molecular basis of chromium insulin interactions. Biochemical and Biophysical Research Communications. 2008, Vol. 369, pp. 725-729.

14 SurTec.http://www.surtec.com.br/

15 RAMEZANZADEH, B., ATTAR, M. M. e FARZAM, M. Corrosion performance of a hotdip-galvanized steel treated by different kinds of conversion coatings. Surface and Coatings Technology. 2010, Vol. 205, pp. 874-884.

16 Lucena, M. P.P. Estudo de otimização dos parâmetros do processo de conversão de revestimentos à base de $\mathrm{Zr}$ e Ti sobre aço galvanizado. Dissertação de Mestrado. Porto Alegre: UFRGS, 2014. 